\title{
Milk Fermented with Lactobacillus casei Strain AP Improves Lipid Profiles in Obese Indonesian Adults
}

\author{
Widodo Widodo ${ }^{1,2, *}$, Anggeria Oktavisa Denta ${ }^{1}$, Sunarti Sunarti ${ }^{1,3}$ and Dietmar Haltrich ${ }^{4}$ \\ 1 Graduate School of Biotechnology, Universitas Gadjah Mada, Yogyakarta 55281, Indonesia; anggie.ok- \\ tavisa@gmail.com (A.O.D.); nartyr@ugm.ac.id (S.) \\ 2 Faculty of Animal Science, Universitas Gadjah Mada, Yogyakarta 55281, Indonesia \\ 3 Department of Biochemistry, Faculty of Medicine Public Health and Nursing, Universitas Gadjah Mada, \\ Yogyakarta 55281, Indonesia \\ 4 Food Biotechnology Laboratory, University of Natural Resource and Life Sciences, 1190 Vienna, Austria; \\ dietmar.haltrich@boku.ac.at \\ * Correspondence: widodohs@ugm.ac.id
}

\begin{abstract}
Obesity and hyperglycemia can trigger various diseases, including diabetes mellitus and cardiovascular ailments. Health-promoting fermented milk products can be useful in tackling these issues. One such product is the fermented milk developed using Lactobacillus casei AP, a probiotic strain from Indonesia that has not been tested in humans thus far. Our objective was to examine the effects of L. casei AP-fermented milk products on lipid profiles, blood glucose levels and monocyte chemoattractant protein-1 (MCP-1) levels in obese adults. A total of 29 obese subjects were given $L$. casei AP-fermented milk products, and their fasting blood glucose, total cholesterol, low-density lipoprotein (LDL), high-density lipoprotein (HDL) and triglyceride levels were measured using diagnostic system kits. MCP-1 levels were measured using enzyme-linked immunosorbent assay. It was determined that the administration of $L$. casei AP-fermented milk products significantly reduced total cholesterol, LDL and triglyceride levels $(p<0.05)$; however, it did not increase HDL $(p>$ $0.05)$, MCP-1 or fasting blood glucose levels ( $p \geq 0.05)$. In conclusion, in obese Indonesian adults, $L$. casei AP-fermented milk might reduce total cholesterol, LDL and triglyceride levels but may not affect HDL, MCP-1 or fasting blood glucose levels.
\end{abstract}

Keywords: Obesity; Lactobacillus casei AP; probiotic; fermented milk; lipid profiles

\section{Introduction}

Obesity exacerbates the risk of several metabolic and degenerative diseases, including cardiovascular ailment, diabetes mellitus, cancer and osteoarthritis [1]. Obesity is multifactorial in origin, and its possible causes include environmental factors and unhealthy behaviours and food selection patterns [1]. Therefore, the development of a variety of functional food products, including dairy products, intended as health foods is urgently needed. The use of probiotic lactic acid bacteria (LAB), which are live microorganisms that confer health benefits to the host when administered in an adequate amount and function as starter bacteria in fermented milk, is an important development in this respect [2]. Maha [3] reported that fermented milk enriched with probiotics reduced hyperglycemia and oxidative stress in diabetic mice. Hsieh et al. [4] showed that in Sprague Dawley rats, oral administration of Lactobacillus reuteri GMNL-263 (Lr263) lowered insulin resistance and blood glucose levels. Sprague Dawley rats fed with a high-fat diet together with the Lactobacillus casei strain Shirota exhibited reduced fat mass and blood glucose and leptin levels, while high-density lipoprotein (HDL) and adiponectin levels were increased when compared with the control group [5]. Blood cholesterol levels were not affected by the oral administration of L. casei in this study [5]. Administration of L. casei CRL 431-fermented milk along with a high-fat diet significantly reduced blood glucose and cholesterol levels in $\mathrm{BALB} / \mathrm{c}$ mice in comparison with the control group, while the addition of this product 
did not affect the serum cholesterol level in mice fed with a normal diet [6]. Yogurt supplemented with antioxidant-rich foods, such as honey, decreased the serum levels of cholesterol, low-density lipoprotein (LDL) and very low-density lipoprotein (VLDL) and increased the HDL levels [7].

L. casei strain AP was previously isolated from the faeces of a breastfed Indonesian infant ( $<1$ month of age) [8]. In Sprague Dawley rats, the consumption of L. casei AP in the form of fermented dairy products reduced hyperglycemia and cholesterol levels [9]. Although $L$. casei AP, a probiotic strain from Indonesia, can be developed into fermented dairy products as a possible health food, it has not been tested in humans thus far.

Hyperglycemia and obesity elevate the level of monocyte chemoattractant protein-1 (MCP-1) [10]. Weight gain is associated with increase in monocyte levels and systemic inflammation, both of which trigger chronic diseases such as cardiovascular ailments and type 2 diabetes mellitus [11]. Because obesity is a harmful condition, our aim was to examine the effects of $L$. casei AP-fermented milk products on the lipid profile and blood glucose and MCP-1 levels in obese Indonesian adults.

\section{Materials and Methods}

\subsection{Preparation of fermented milk}

For the preparation of fermented milk, $2 \%$ skimmed milk was added to fresh cow's milk; the mixture was pasteurised at $80^{\circ} \mathrm{C}$ for $30 \mathrm{~min}$ and then cooled down to $45^{\circ} \mathrm{C}$. The pasteurised milk was inoculated with $5 \%(\mathrm{v} / \mathrm{v})$ bulk starter stock of $L$. casei AP culture and then incubated at room temperature $\left(37^{\circ} \mathrm{C}\right)$ for $18 \mathrm{~h}$.

\subsection{Study design and subject selection}

An experimental study involving pre- and post-tests was designed in a group of obese adults. The inclusion criteria were as follows: body mass index (BMI) >25; age between 18 and 45 years; and willingness to consume L. casei AP probiotic-fermented milk product. The exclusion criteria were as follows: intake of antibiotics; consumption of medications such as weight loss drugs; lactose intolerance; digestive tract disorders; and pregnancy or breastfeeding. A total of 29 study participants were requested to provide written informed consent to undergo anthropometry and blood pressure measurements and complete the research questionnaires.

\subsection{Treatment and laboratory investigations}

All subjects consumed $100 \mathrm{~mL}$ of $L$. casei AP-fermented milk product per day for a duration of 4 weeks. The fasting serum levels were sampled before and after consumption of the product. A total of $10 \mathrm{~mL}$ of blood was withdrawn from each subject's vein using a 10-mL syringe (Terumo). The collected blood was placed in tubes containing ethylenediaminetetraacetic acid (BD Vacutainer) and centrifuged (Centrifuge $5430 \mathrm{R}$ ) at $3500 \mathrm{~g}$ for 15 min for plasma separation. The levels of fasting blood glucose, total cholesterol, LDL, HDL and triglycerides were measured using Diasys Diagnostic Systems kits in accordance with the manufacturer's protocols. A wavelength of $500 \mathrm{~nm}$ was employed for the spectrophotometric (Hitachi, U-1800) determination of absorbance in each lipid profile test. The level of MCP-1 was measured using enzyme-linked immunosorbent assay (ELISA, Fine Test Kits) in accordance with the kit manual protocol. The test results were read using a microplate reader (Zenix-320) at $450 \mathrm{~nm}$ to determine the absorbance (optical density).

\subsection{Statistical analysis}

Data pertaining to the effects of fermented milk administration on the levels of MCP1 , fasting blood glucose and lipid profiles were analysed; blood pressure and anthropometry of the study subjects were compared using paired t-test and Wilcoxon test with SPSS version 16.0 software.

\section{Results}




\subsection{Patients}

Table 1 displays the characteristics of the 29 study participants, of which 15 were men (51.7\%) and 14 were women (48.3\%). Their mean age, weight and BMI were $25.1 \pm 4.1$ years, $85.3 \pm 16.4 \mathrm{~kg}$ and $32.9 \pm 5.4 \mathrm{~kg} / \mathrm{m}^{2}$, respectively. Weight, BMI, hip circumference and blood pressure decreased slightly after the consumption of milk products fermented with $L$. casei AP. However, there were no differences in waist circumference before and after the treatment.

Table 1. Anthropometric measurements, blood pressure and other characteristics of the research subjects.

\begin{tabular}{|c|c|c|c|c|}
\hline Subject characteristics $(n=29)$ & Mean \pm SD or $n(\%)$ & Before treatment & After treatment & $p$ value \\
\hline Age (year) & $25.1 \pm 4.1$ & - & - & - \\
\hline Gender & - & - & - & - \\
\hline Male, $n(\%)$ & $15(51.7 \%)$ & - & - & - \\
\hline Female, $n(\%)$ & $14(48.3 \%)$ & - & - & - \\
\hline Weight $(\mathrm{kg})$ & - & $85.3 \pm 16.4$ & $84.7 \pm 16.4$ & $0.000{ }^{a *}$ \\
\hline Body mass index $\left(\mathrm{kg} / \mathrm{m}^{2}\right)$ & - & $32.9 \pm 5.4$ & $32.4 \pm 4.6$ & $0.004^{b *}$ \\
\hline Waist circumference $(\mathrm{cm})$ & - & $97.1 \pm 13$ & $97.4 \pm 13.3$ & $0.631 \mathrm{a} / \mathrm{ns}$ \\
\hline Hip circumference $(\mathrm{cm})$ & - & $112.8 \pm 10.5$ & $111.4 \pm 9.9$ & $0.003 a^{*}$ \\
\hline Blood pressure (mmHg) & - & - & - & - \\
\hline Systole & - & $115.2 \pm 11$ & $104.7 \pm 9.8$ & $0.000 a^{*}$ \\
\hline Diastole & - & $74.3 \pm 8$ & $67.9 \pm 7.3$ & $0.003^{\mathrm{b} *}$ \\
\hline
\end{tabular}

${ }^{*}$ significant, $p<0.05, \mathrm{~ns}=$ not significant, $p>0.05 .{ }^{\mathrm{a}}$ paired t-test, ${ }^{\mathrm{b}}$ Wilcoxon test

\subsection{The effect of L. casei AP-fermented milk products on fasting blood glucose levels in obese adults}

The decrease in fasting blood glucose levels following the consumption of milk fermented with L. casei strain AP was not significant $(p>0.05)$ (Figure 1$)$. This observation is in contrast to the findings of Widodo et al. [9], who showed that the administration of milk containing L. casei AP for 15 days significantly reduced the level of blood glucose ( $p$ $<0.05$ ) in Sprague Dawley rats fed with a high-fat and high-fructose diet. This discrepancy might be due to the fact that the rats were conditioned to have hyperglycemia. The administration of milk products fermented with $L$. casei AP to humans with normal blood glucose levels did not significantly decrease the levels $(p>0.05)$. In agreement with our findings, Prakash et al [12]. observed that the administration of pasteurised milk products that were fermented with Lactobacillus bulgaricus (UBLB-38) and Streptococcus thermophilus (UBST-50) to humans with normal blood glucose levels did not result in significant differences.

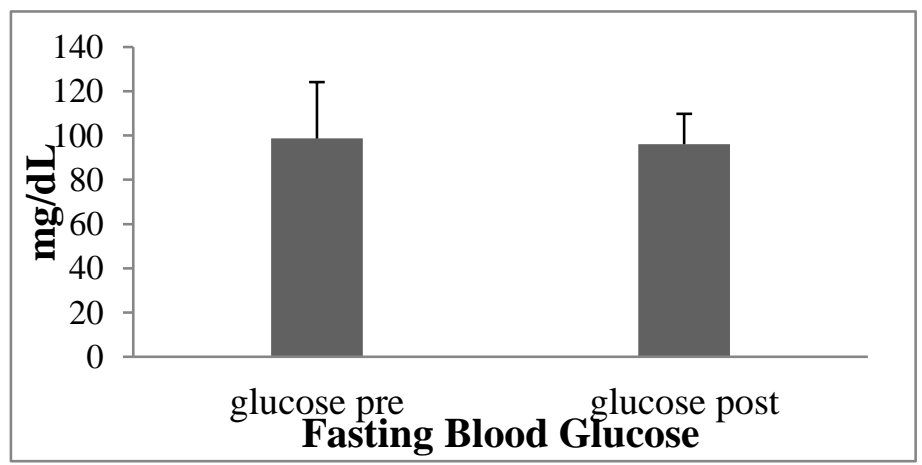

Figure 1. Effect of milk products fermented with Lactobacillus casei AP on the fasting blood glucose level of obese adults.

\subsection{The effect of L. casei AP-fermented milk products on MCP-1 levels in obese adults}

The level of MCP-1 showed a non-significant decrease $(p>0.05)$ after the consumption of milk fermented with $L$. casei strain AP, which could be attributed to the fact that all the study subjects had normal blood glucose levels (i.e., they were not hyperglycaemic) 
(Figure 2). As reported previously, MCP-1 expression may be influenced by various stimuli, including hyperglycaemia [13].

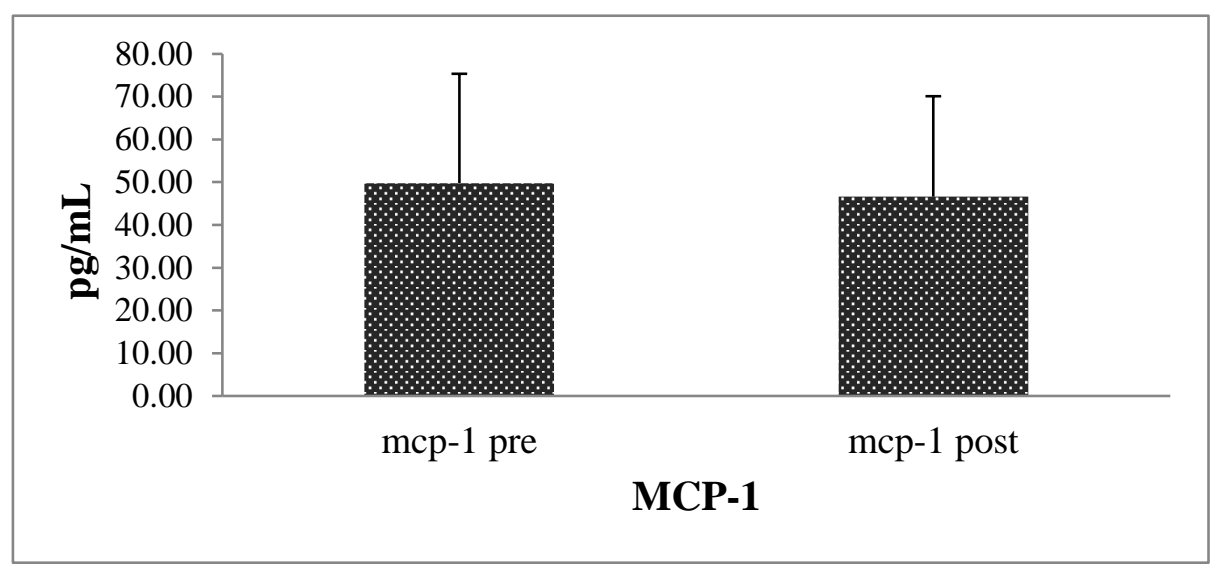

Figure 2. Effect of milk products fermented with Lactobacillus casei AP on the MCP-1 levels preand post-treatment.

\subsection{The effect of L. casei AP-fermented milk products on lipid profiles in obese adults}

Total cholesterol, LDL and triglyceride levels decreased significantly to normal levels $(p<0.05$, Figure 3$)$ after the consumption of milk products fermented with L. casei AP, but the increase in HDL levels was not significant $(p>0.05)$. In this study, we based the normal lipid profile values on the report by Ma and Shieh [14] $(<200 \mathrm{mg} / \mathrm{dL}$ for total cholesterol, $<100 \mathrm{mg} / \mathrm{dL}$ for LDL and $<150 \mathrm{mg} / \mathrm{dL}$ for triglycerides).

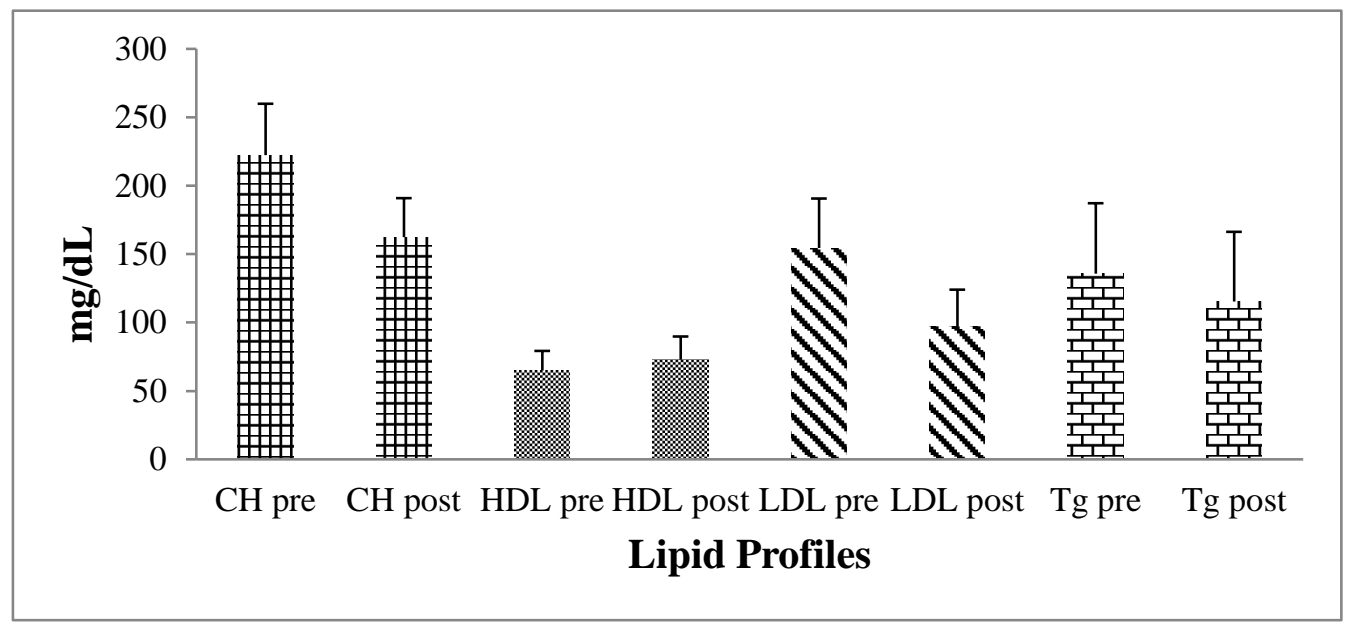

Figure 3. Effects of milk products fermented with Lactobacillus casei AP on the lipid profiles preand post-treatment. $\mathrm{CH}$, cholesterol; HDL, high-density lipoprotein; LDL, low-density lipoprotein; $\mathrm{Tg}$, triglycerides

We observed increased HDL levels after the consumption of the fermented milk (Figure 3), although the increase was not significant. According to previous studies, HDL levels of $\geq 60 \mathrm{mg} / \mathrm{dL}$ can offer protection against heart diseases $[14,15,16]$. Other meta-analyses of randomised controlled trials have shown that probiotics do not significantly influence the HDL levels. Another systematic review and meta-analysis of randomised controlled trials by Wu et al. [17] demonstrated the non-significant effects of probiotics on HDL levels.

\section{Discussion}

Yogurt supplemented with antioxidant-rich foods, such as honey, has been reported to control hypercholesterolemia, a risk factor of atherosclerosis, by reducing the serum levels of cholesterol, LDL and VLDL and increasing the HDL levels [7]. Fermented milk 
that contains the probiotics Bifidobacterium and Lactobacillus gesseri has been proven to reduce total cholesterol, LDL and VLDL-C and increase HDL levels $[15,18]$. The milk components hydroxymethyl glutarate, uronic acid and conjugated linoleic acid (CLA) play pertinent roles in lowering the cholesterol level [19]. Ogawa et al. [20] found that LAB fermentation is effective in improving the level of CLA. L. casei AP is an LAB that can produce short-chain fatty acids (SCFA) [21]. In addition to CLA, SCFA lowers lipolysis in the adipose tissue, thereby reducing the levels of circulating free fatty acids and non-esterified fatty acids [22]. Subsequently, low levels of circulating free fatty acids curb triglyceride synthesis in the liver and reduce VLDL secretion, thereby controlling the surge in plasma triglyceride levels [23]. Similarly, Canfora et al. [24] identified that SCFA inhibits lipolysis in the adipose tissue and reduces the circulating triglyceride levels. Likewise, Gao et al. [25] noted that butyrate, an SCFA obtained through fermentation by certain intestinal bacteria, improves insulin sensitivity and inhibits lipolysis. Using a human enterocyte cell line (Caco-2/TC-7), Alvaro et al. [26] studied the effect of SCFA and asserted that butyrate and propionate inhibit the expression of nine key genes in cholesterol biosynthesis. Probiotics assimilate cholesterol and deconjugate bile salts by producing bile salt hydrolase (BSH) and reducing the blood cholesterol levels [27]. In particular, administration of BSH-active L. acidophilus CHO-220 combined with the prebiotic inulin significantly reduces plasma total cholesterol and LDL-C levels [27].

Hike in MCP-1 expression is modulated by the production of reactive oxygen species, which is triggered by high blood glucose levels [28]. In this study, because blood glucose levels were normal, MCP-1 levels were not high; consequently, the reductions were not significant. As previously reported by Bassaganya-Riera et al. [29] the presence of CLA produced by probiotics might contribute to the suppression of MCP-1 expression in colonic lamina propria cells. High glucose levels lead to the activation of protein kinase $C$, triggering the production of reactive oxygen species by increasing NADPH oxidase and p66 shc adapter proteins.

Probiotics reduce blood glucose levels by activating pancreatic $\beta$-cells, thereby playing a significant role in maintaining blood glucose homeostasis for insulin production [30]. Yogurt with added honey can be used as an anti-hyperglycaemic agent by suppressing the absorption and the inhibition of glucose by $\alpha$-glucosidase along with the activation of antioxidants that restore the functioning of the pancreatic $\beta$-cells [7]. Probiotics have been reported to exert anti-diabetic effects on insulin resistance in type 2 diabetes mellitus patients by increasing the number of natural killer T-cells, significantly reducing the fasting blood glucose levels [31].

Further research is needed to examine the effects of L. casei AP-fermented milk products on patients with type 2 diabetes mellitus and non-obese controls. We anticipate that the findings of this study will support the development of fermented dairy products as functional foods. These products are also expected to play a key role in the prevention of type 2 diabetes mellitus and cardiovascular diseases, both of which obese people are at a high risk of developing.

\section{Limitations}

We designed an experimental study with pre- and post-tests in one group of obese adults. A major limitation of our study is that we did not normalise the data to a similarly treated non-obese group.

\section{Conclusions}

Our results have demonstrated, for the first time, the beneficial effects of consuming L. casei AP probiotic-fermented milk on the lipid profile of obese Indonesian adults. Consumption of the fermented milk product over a 4-week period reduced total cholesterol, LDL and triglyceride levels but had no effect on HDL, MCP-1 or fasting blood glucose levels. Further research is needed to examine the effects of L. casei AP-fermented milk products on patients with type 2 diabetes mellitus. 
Author Contributions: Conceptualization, W.W. and S.S.; Data curation, A.O.D.; Formal analysis, W.W. and D.H.; Funding acquisition, W.W. and A.O.D.; Investigation, S.S.; Methodology, W.W., A.O.D., S.S. and D.H.; Supervision, W.W.; Validation, S.S, and D.H.; Visualization, S.S.; Writing original draft, W.W. and A.O.D.; Writing - review \& editing, W.W. and D.H.

Funding: This research was funded by Indonesian Ministry for Research and Higher Education, grant number 1850/UN1/DITLIT/DIT.LIT/LT/2018.

Institutional Review Board Statement: The study was conducted in accordance with the Declaration of Helsinki and the protocol was approved by Medical and Health Research Ethics Committee of the Faculty of Medicine Gadjah Mada University-DR Sardjito General Hospital with reference number: KE/FK/0809/EC/2017.

Informed Consent Statement: Informed consent was obtained from all subjects involved in the study.

Data Availability Statement: All data generated or analysed during this study have been included in the paper. The raw data are available from the corresponding author on reasonable request.

Conflicts of Interest: The authors declare no conflict of interest.

\begin{tabular}{ll} 
Abbreviations & \\
\hline BSH & Bile salt hydrolase \\
\hline CLA & Conjugated linoleic acid \\
\hline HDL & High-density lipoprotein \\
\hline LAB & Lactic acid bacteria \\
\hline LDL & Low-density lipoprotein \\
\hline SCFA & Short-chain fatty acids \\
\hline VLDL & Very low-density lipoprotein \\
\hline
\end{tabular}

\section{References}

1. Lyssenko, V.; Jonsson, A.; Almgren, P.; Pulizzi, N.; Isomaa, B.; Tuomi, T.; Berglund, G.; Altshuler, D.; Nilson, P.; Groop, 1. Clinical risk factors, DNA variants, and the development of type 2 diabetes. N. Engl J. Med. 2008, 359, 2220-2232, doi:10.1056/NEJMoa0801869.

2. Widodo, W.; Handaka, R.; Wahyuni, E.; Taufiq, T.T. The quality of milk produced using intestinal-origin lactic acid bacteria as starters. Int. Food Res. J. 2017, 24, 2371-2376.

3. Maha, B. Antidiabetic potential of turmeric with/without fermented milk enriched with probiotics in diabetic rats. Am. J. Biomed Life Sci. 2013, 1, 1-7, doi:10.11648/j.ajbls.20130101.11.

4. Hsieh, F-C.; Lee, C-L.; Chai, C-Y.; Chen, W-T.; Lu, Y-C.; Wu, C-S. Oral administration of Lactobacillus reuteri GMNL-263 improves insulin resistance and ameliorates hepatic steatosis in high fructose-fed rats. Nutr Metab. 2013, 10,35, doi:10.1186/17437075-10-35.

5. Karimi, G.; Sabran, M.R.; Jamaluddin, R.; Parvaneh, K.; Mohtaruddin, N.; Ahmad, Z.; Khazaai, H.; Khodavandi, A. The antiobesity effects of Lactobacillus casei strain Shirota versus orlistat on high fat diet-induced obese rats. Food Nutr Res. 2015, 59, 29273, doi:10.3402/fnr.v\%25v.29273.

6. Nunez, I.N.; Galdeano, C.M.; de LeBlanc, A.D.M.; Perdigon, G. Evaluation of immune response, microbiota, and blood markers after probiotic bacteria administration in obese mice induced by a high-fat diet. Nutrition 2014, 30, 1423-1432, doi:10,1016/j.nut.2014.03.025.

7. Bukhari, H.M.; Abdelghany, A.H.; Nada, I.S.; Header, E.A. Effect of yoghurt pillared with propolis on hyperglycemic rats. Egypt J. Hosp Med. 2012, 49, 691-704, doi:10.12816/EJHM.2012.16208.

8. Widodo, W.; Anindita, N.S.; Taufiq, T.T.; Wahyuningsih, T.D. Identification of Pediococcus strains isolated from feces of Indonesian infants with in vitro capability to consume prebiotic inulin and to adhere on mucus. Indones J. Biotechnol. 2012, 17, 132143, doi:10.22146/ijbiotech.7859.

9. Widodo, W.; Harsita, P.A.; Sukarno, A.S.; Nurrochmad, A. Antidiabetic effect of milk fermented using intestinal probiotics. Food Sci. Nutr. 2019, 49, 1063-1074, doi:10.1108/NFS-11-2018-0326.

10. Panee, J. MCP-1 in obesity and diabetes. Cytokine, 2012, 60, 1-12, doi:10.1016/j.cyto.2012.06.018.

11. Breslin, W.L.; Johnston, C.A.; Strohacker, K.; Carpenter, K.C.; Davidson, T.R.; Moreno, J.P.; Foreyt, J.P.; McFarlin, B.K. Obese Mexican American children have elevated MCP-1, TNF- $\alpha$, monocyte concentration and dyslipidemia. Pediatrics. 2015, 129, e1180-6, doi:10.1542/peds.2011-2477.

12. Prakash, M.S.; Rao, P.A.; Chathyushya, K.B.; Hariacharan, G.; Madhavi, G.; Sumalata, G.; Sravanthi, M.; Mounika, M.; Tejaswi, P.S.; Hemalatha, R. Effect of supplementation on the fasting blood glucose and immune status in type II diabetes mellitus. Imp J. Interdis Res. 2017, 3, 1244-1248. 
13. Chen, B.; He, T.; Xing, Y.; Cao, T. Effect of quercetin on the expression of MCP-1, MMP-9 and VEGF in rats with diabetic retinopaty. Exp Ther Med. 2017, 14, 6022-6026, doi:10.3892/etm.2017.5275.

14. Ma, H.; Shieh, K.J. Cholesterol and human health. J. Am. Sci. 2006, 2, 46-50.

15. Pereira, D.I.A.; Gibson, G.R. Cholesterol Assimilation by Lactic Acid Bacteria and Bifidobacteria Isolated from the Human Gut. Appl. Environ. Microbiol. 2002, 68, 4689-4693, doi:10.1128/aem.68.9.4689-4693.2002.

16. Cho, Y.A.; Kim, J. Effect of probiotics on blood lipid concentrations. Medicine (Baltim) 2015, 94, 1-10, doi:10.1097/MD.0000000000001714.

17. Wu, Y.; Zhang, Q.; Ruan, Z. Effect of probiotic lactobacillus on lipid profile: a systematic review and meta-analysis of randomized controlled trial. PLoS One, 2017, 12, e0178868, doi:10.1371/journal.pone.0178868.

18. Kang, J.H.; Yun, S.I.; Park, H.O. Effects of Lactobacillus gasseri BNR17 on body weight and adipose tissue mass in diet-induced overweight rats. J. Microbiol. 2010, 48, 712-714, doi:10.1007/s12275-010-0363-8.

19. Kim, Y.J.; Liu, R.H. Increase of conjugated linoleic acid content in milk by fermentation with lactic acid bacteria. J. Food Sci. 2002, 67, 1731-1737, doi:10.1111/j.1365-2621.2002.tb08714.x.

20. Ogawa, J.; Kishino, S.; Ando, A.; Sugimoto, S.; Mihara, K.; Shimizu, S. Production of conjugated fatty acid by lactic acid bacteria. J. Biosci. Bioeng. 2005, 100, 355-364, doi:10.1263/jbb.100.355.

21. Kusmiyati, N.; Wahyuningsih, T.D.; Widodo, W. Prebiotic effect of inulin extract from Dahlia tubers (Dahlia pinnata L.) on the growth performance of intestinal-origin Lactobacillus casei AP. Pak J. Nutr. 2018, 17, 405-410, doi:10.3923/pjn.2018.405.410.

22. Marinangeli, C.P.F.; Peter, J.H.J. Plant sterols, marine-derived omega-3 fatty acids and other functional ingredients: a new frontier for treating hyperlipidemia. Nutr Metab. 2010, 7, 76, doi:10.1186/1743-7075-7-76.

23. Taskinen, M.R. Type 2 diabetes as a lipid disorder. Curr. Mol. Med. 2005, 5, 297-308, doi:10.2174/1566524053766086.

24. Canfora, E.E.; Jocken, J.W.; Blaak, E.E. Short-chain fatty acids in control of body weight and insulin sensitivity. Nat. Rev. Endocrinol. 2015, 11, 577-592, doi:10.1038/nrendo.2015.128.

25. Gao, Z.; Yin, J.; Zhan,g J.; Ward, R.E.; Martin, R.J.; Lefevre M.; Cefalu, W.T.; Ye, J. Butyrate improves insulin sensitivity and increases energy expenditure in mice. Diabetes 2009, 58, 1509-1517, doi:10.2337/db08-1637.

26. Alvaro, A.; Solà, R.; Rosales, R.; Ribalta, J.; Anguera, A.; Masana, L.; Vallve, J.C. Gene expression analysis of a human enterocyte cell line reveals downregulation of cholesterol biosynthesis in response to short-chain fatty acids. IUBMB Life 2008, 60, 757-764, doi:10.1002/iub.110.

27. Jones, M.L.; Tomaro-Duchesneau, C.; Martoni, C.J.; Prakash, S. Cholesterol lowering with bile salt hydrolase-active probiotic bacteria, mechanism of action, clinical evidence, and future direction for heart health applications. Expert Opin Biol. Ther. 2013, 13, 631-642, doi:10.1517/14712598.2013.758706.

28. Paneni, F.; Beckman, J.A.; Creager, M.A.; Cosentino, F. Diabetes and vascular disease: pathophysiology, clinical consequences and medical therapy: part I. Eur Heart J. 2013, 34, 2436-2443, doi:10.1093/eurheartj/eht149.

29. Bassaganya-Riera, J.; Viladomiu, M.; Pedragosa, M.; De Simone, C.; Carbo, A.; Shaykhutdinov, R.; Jobin, C.; Arthur, J.C.; Corl, B.A.; Vogel, H.; Storr, M.; Hontecillas, R. Probiotic bacteria produce conjugated linoleic acid locally in the gut that targets macrophage PPAR $\gamma$ to suppress colitis. PLoS One 2012, 7, 1-8, doi:10.1371/journal.pone.0031238.

30. Hirahatake, K.M.; Slavin, J.L.; Maki, K.C.; Adams, S.H. Association between dairy foods, diabetes and metabolic health: potential mechanisms and future directions. Metabolism 2014, 63, 618-627, doi:10.1016/j.metabol.2014.02.009.

31. Zhang, Q.; Wu, Y.; Fei, X. Effect of probiotics on glucose metabolism in patient with type 2 diabetes mellitus: a meta-analysis of randomized controlled trial. Medicina 2016, 52, 28-34, doi:10.1016/j.medici.2015.11.008. 